\title{
Aspectos biológicos de Pelidnota fulva Blanchard, 1850 (Coleoptera, Scarabaeidae, Rutelinae)
}

\author{
Sérgio Roberto Rodrigues ${ }^{1,2}$ \& Josani da Silva Falco ${ }^{1}$ \\ ${ }^{1}$ Universidade Estadual de Mato Grosso do Sul - UEMS, \\ Rod. Aquidauana/CERA, Km 12, CEP 79200-000, Aquidauana, MS, Brasil \\ ${ }^{2}$ Autor para correspondência: Sérgio Roberto Rodrigues, e-mail: sergio@uems.br
}

RODRIGUES, S.R. \& FALCO, J.S. Biological aspects of the Pelidnota fulva Blanchard, 1850 (Coleoptera, Scarabaeidae, Rutelinae). Biota Neotrop. 11(1): http://www.biotaneotropica.org.br/v11n1/en/ abstract?article+bn02311012011.

\begin{abstract}
Some species of Scarabaeidae present an important function in the decomposition and recycle of organic material and because there is little information on this group of insects, the objective of the present study was to evaluate the biological aspects of Pelidnota fulva Blanchard, 1850. Determination of the period of occurrence was performed by capture of adults with a light trap and in laminated areas of the experimental farm belonging to the Universidade Estadual de Mato Grosso do Sul, Brazil, from January 2008 to December 2009. After mating and obtaining of the eggs biological studies were initiated, in which the insects were maintained in an acclimatized chamber at $26+1{ }^{\circ} \mathrm{C}$ with photophate for 12 hours. Adult occurrence was short in the field, being captured in October and November of 2008 and September and October of 2009. Eggs were obtained in the laboratory which possessed average dimensions of $2.5 \times 1.9 \mathrm{~mm}$, and after a few days the same dimensions averages $3.4 \times 3.3 \mathrm{~mm}$. The embryonic period lasted 15.1 days; the first, second and third instar larvae periods lasted 22.4, 36.9 and 209.7 days, respectively; and the pupa phase lasted 20.7 days. Average duration of the egg to adult cycle was 309.3 days, characterizing the species as univoltine. Adults possess longevity of 12.8 days. Mating was observed in the laboratory which presented minimal and maximal durations of 8 minutes and 1 hour and 13 minutes.

Keywords: biological cycle, population dynamics, Rutelinae, Scarabaeoidea.
\end{abstract}

RODRIGUES, S.R. \& FALCO, J.S. Aspectos biológicos de Pelidnota fulva Blanchard, 1850 (Coleoptera, Scarabaeidae, Rutelinae). Biota Neotrop. 11(1): http://www.biotaneotropica.org.br/v11n1/pt/ abstract?article+bn02311012011.

Resumo: Algumas espécies de Scarabaeidae apresentam importante função na decomposição e reciclagem de material vegetal e como existem poucas informações sobre esse grupo de insetos, o presente trabalho teve por objetivo estudar os aspectos biológicos de Pelidnota fulva Blanchard, 1850. A determinação do período de ocorrência foi realizada com coletas de adultos com armadilha luminosa e em fontes luminosas na fazenda experimental da Universidade Estadual de Mato Grosso do Sul de janeiro de 2008 a dezembro de 2009. Com a formação de casais e obtenção de ovos foram iniciados os estudos de biologia, sendo os insetos mantidos em câmara climatizada a $26+1{ }^{\circ} \mathrm{C}$, com fotofase de 12 horas. Os adultos ocorreram durante curto período de tempo em campo, pois foram coletados em outubro e novembro de 2008, e em setembro e outubro em 2009. Em laboratório foram obtidos ovos, os quais possuem dimensões médias 2,5×1,9 mm, e após alguns dias atingem as dimensões médias de 3,4 ×3,3 mm. O período embrionário durou 15,1 dias, as larvas de primeiro instar duraram 22,4 dias, as de segundo instar 36,9 dias e as de terceiro 209,7 dias, a fase de pupa durou 20,7 dias. A duração média do ciclo de ovo a adulto foi de 309,3 dias, caracterizando a espécie como univoltina. Os adultos possuem longevidade de 12,8 dias. Foram observadas cópulas no laboratório que duraram no mínimo oito minutos e no máximo 1 hora e 13 minutos.

Palavras-chave: ciclo biológico, dinâmica populacional, Rutelinae, Scarabaeoidea. 


\section{Introdução}

Os Scarabaeidae apresentam grande importância para o meio ambiente onde se desenvolvem, pois as larvas de várias espécies se nutrem de material vegetal em decomposição, proporcionando a reciclagem de nutrientes. O mesmo acontece com as larvas de algumas espécies de Pelidnota MacLeay, 1819 (Morón 1976, Morón \& Deloya 2002).

Flores et al. (2008) ao estudarem os Scarabaeidae de Villaflores, Xiapas, no México verificaram que os representantes de Pelidnota apresentam hábito xilofitófago. Neita et al. (2006) obtiveram larvas de Pelidnota chiriquina Bates, 1904 em troncos em decomposição de Brosimum utile (Kunt) (Moraceae) na Colômbia.

Hayes (1925) verificou que os adultos de Pelidnota punctata (Linn. 1758) alimentam-se de folhas de videiras, enquanto que as larvas desenvolvem-se em troncos caídos de árvores e sua ação acelera a decomposição do material vegetal. Os adultos dessa espécie segundo Buss (2006) são atraídos também por fontes luminosas na Flórida.

Para Pelidnota (Pelidnota) virescens Burmeister, 1844, Morón \& Deloya (2002) realizaram a criação de larvas com fragmentos de madeira de Annona sp. (Annonaceae) em decomposição, e a larva de terceiro instar foi descrita por Morón (1976) no México. Os adultos de $P$. virescens alimentam-se de folhas de Parkinsonia aculeata (Fabaceae) e Baccharis salicifolia (Asteraceae) (Garcia et al. 2009).

Com armadilha luminosa Pardo-Locarno et al. (2005) coletaram Pelidnota prasina (Germar 1824), na Colômbia e Carrilo Ruiz \& Morón (2003) coletaram Pelidnota strigosa Laporte, 1840 no México. Delgado-Castillo et al. (1988) relacionaram várias espécies de Pelidnota ocorrentes no México.

Paucar-Cabrera (2005) ao realizar a revisão da subfamília Rutelinae no Equador relacionou 22 espécies de Pelidnota ocorrendo nesse país. Maes et al. (1997) relacionaram 18 novas espécies de Scarabaeidae para a fauna da Nicarágua, incluindo Pelidnota hirsutiphallica Ratcliffe \& Jameson, 1989. No Panamá, Ratcliffe (2002) e Hardy (1975) relacionam várias espécies de Pelidnota ocorrentes.

No Brasil poucos são os estudos conhecidos sobre os representantes de Pelidnota. Lordello (1951) coletou em Cordeirópolis, SP, adultos de Pelidnota pallidipennis Bates, 1904 alimentando-se de flores de plantas de roseira. Adultos de Pelidnota unicolor (Drury,1778), P. sumptuosa (Vig., 1825) e P. testaceovirens Blanchard, 1850 foram coletados em flores de Matayba guianensis Aubl. (Sapindaceae) em área de cerrado em Uberlândia, MG (Carvalho 2009). Silva et al. (1968) informam que adultos de Pelidnota aeruginosa L., 1758 alimentam-se de folhas de videira e flores e broto de vimeiro no Rio Grande do Sul, P. pallidipennis Bates, 1904 alimenta-se de folhas e flores de roseiras e açoita cavalo na BA, GO, MG, MT e SP, P. rugulosa Burmeister, 1844 consome folhas de Cassia sp., P. sordida Germar, 1824 alimenta-se de folhas e flores de roseira no RJ, P. sumptuosa (Vigors, 1825) alimenta-se de folhas e flores de roseira em SP e P. zikani Ohaus, 1922 alimenta-se de frutos de brinco de princesa em MG e RJ.

Bione et al. (2005) para realizarem a descrição do cariótipo de P. pallidipennis efetuaram coletas de adultos na floresta atlântica, no nordeste Brasileiro no Estado de Pernambuco próximo à Igarassú. Em Santa Bárbara, MG, com o uso de armadilhas luminosas, Freitas et al. (2002) coletaram em áreas de Eucalyptus grandis W. Hill ex Maiden um adulto de Pelidnota aeruginosa (L., 1758).

Face aos poucos estudos relacionando os Scarabaeidae que auxiliam no processo de decomposição de materiais vegetais, foram desenvolvidos estudos dos aspectos biológicos de Pelidnota fulva Blanchard, 1850 em Aquidauana, MS, a qual ocorre na Argentina, Bolívia, Paraguai e Brasil (Howden 1998, Blackwelder 1944), enquanto que Viana et al. (2001) obtiveram adultos dessa espécie com armadilha luminosa em Cruz das Almas, BA.

\section{Material e Métodos}

Os estudos foram desenvolvidos na Universidade Estadual de Mato Grosso do Sul, no município de Aquidauana, MS. De janeiro de 2008 a dezembro de 2009, adultos de Pelidnota fulva foram coletados diariamente com uso de armadilha luminosa, modelo "Luiz de Queiroz", instalada entre área de pastagem e culturas e quando começaram a ocorrer, iniciavam-se as observações no início do escurecer sob postes de luz, para coleta de adultos, tendo em vista a atratividade destes por fontes luminosas. Os adultos obtidos foram conduzidos para laboratório para observações de comportamento, e início dos estudos dos aspectos biológicos como descrito por Rodrigues et al. (2008b).

Foram formados 12 casais de $P$. fulva os quais permaneceram em seis recipientes de plástico $(20 \times 30 \times 12 \mathrm{~cm})$ contendo solo, material orgânico (restos vegetais em decomposição e pedaços de madeira) e húmus para que realizassem a cópula e oviposição. Cada um dos recipientes contendo dois machos e duas fêmeas, e envoltos com tecido de voal para contenção dos insetos, foram vistoriados semanalmente quanto à presença de ovos, os quais foram utilizados para dar início aos estudos de biologia.

Os ovos foram acondicionados em placas de Petri, sobre uma camada de solo e mantidos em câmara climatizada $\left(26 \pm 1^{\circ} \mathrm{C}\right.$ e 12 horas de fotofase) e a cada três dias vistoriava-se o material para registro da duração do período embrionário.

Com a eclosão das larvas, estas eram individualizadas e transferidas para recipientes de plástico de $500 \mathrm{~mL}$, contendo solo, material vegetal em decomposição (restos vegetais em decomposição e pedaços de madeira) e húmus, onde formam criadas até a emergência dos adultos. A duração dos instares larvais e da pupa, foram registrados, bem como a longevidade dos adultos. A largura da cápsula cefálica de 10 larvas foi mensurada em intervalos de 3 a 4 dias para acompanhar o crescimento, segundo a metodologia adaptada de Rodrigues et al. (2008ab).

Alguns espécimes foram depositados na coleção de entomologia da Universidade Estadual de Mato Grosso do Sul em Aquidauana, MS.

\section{Resultados e Discussão}

Ao se obter os adultos de $P$. fulva, foi possível definir a época de ocorrência destes no campo, assim, em 2008 foram coletados 27 adultos em outubro e quatro em novembro, enquanto que em 2009 um adulto foi coletado em setembro e em outubro 22. Em 2008 dos 31 adultos coletados 26 eram machos e cinco fêmeas, e em 2009, dos 23 coletados, 14 eram machos e nove fêmeas, sendo os machos os mais atraídos pelas fontes luminosas (Figura 1).

De setembro a dezembro normalmente as temperaturas e umidade na região de Aquidauana são altas, o que provavelmente favorece a ocorrência e reprodução dessa espécie, tanto que adultos trazidos para o laboratório nos dois anos de estudos, ovipositaram durante o mês de outubro e novembro.

Foram observados adultos em cópula durante os períodos da manhã $(n=3)$, tarde $(n=4)$ e noite $(n=1)$. A cópula durou em média 21 minutos e 25 segundos $(\mathrm{n}=8)$, no mínimo oito minutos e no máximo 1 hora e 13 minutos. Em algumas cópulas foi observado que após o término o macho não se soltava da fêmea $(\mathrm{n}=5)$, permanecia sobre essa em média de 21 minutos, e em outras ocasiões após a cópula o macho se soltava da fêmea $(\mathrm{n}=3)$. Rodrigues et al. (2008b) verificaram que machos de Liogenys fuscus Blanchard, 1851 permanecem cerca de 40 minutos com as fêmeas após o término da cópula. 
Durante uma das observações de cópula, o macho e a fêmea de $P$. fulva se enterraram no solo dentro dos recipientes plásticos. Para P. fulva criada em laboratório não houve antes da cópula, nenhuma evidência de atividade de chamamento ou de possível liberação de hormônio para atração dos sexos. Rodrigues et al. (2008b) verificaram em adultos de L. fuscus comportamento que indicava a presença e possível liberação de hormômio sexual.

Uma fêmea dissecada em outubro de 2008, apresentou 32 ovos em desenvolvimento, e em laboratório fêmeas ovipositaram no máximo 20 ovos $(n=4)$. A partir dos adultos mantidos em laboratório foram obtidos ovos, os quais deram início aos estudos. Os ovos recém depositados possuem dimensões médias de 2,5 × 1,9 mm, são colocados no solo em câmaras com dimensões médias de $3,4 \times 3,3 \mathrm{~mm}(\mathrm{n}=10)$. Os ovos de Leucothyreus dorsalis Blanchard, 1850 e Cyclocephala verticalis Burmeister, 1847 também são depositados em câmaras no solo, segundo Rodrigues et al. (2010a, b), da mesma forma como observado para P. fulva.

Alguns dias após a oviposição observou-se aumento nas dimensões dos ovos os quais passam a medir em média $3,1 \times 2,5 \mathrm{~mm}$ $(\mathrm{n}=9)$. Para $P$. punctata os ovos possuem dimensões de $2,0 \times 1,5 \mathrm{~mm}$, e também aumentam de tamanho segundo Hoffmann (1936). Morón \& Deloya (2002) indicaram que os ovos de $P$. virescens possuem dimensões de 1,3 x $0,9 \mathrm{~mm}$.

O período embrionário de $P$. fulva durou 15,1 dias (Tabela 1) enquanto que para $P$. punctata o período embrionário durou em média 14,9 dias, quando criado a $25^{\circ} \mathrm{C}$ (Hayes 1925) e para $P$. virescens durou em média 24 dias, quando criado a $29^{\circ} \mathrm{C}$ (Morón \& Deloya 2002). Portanto, as durações do período embrionário de $P$. fulva e P. punctata são semelhantes.

As larvas de primeiro instar de P. fulva duraram 22,4 dias, as de segundo instar 36,9, as de terceiro instar 209,7 e a pupa 20,7 dias (Tabela 1). O primeiro e segundo instares possuem curta duração, quando comparados com o terceiro instar que dura quase sete meses. As larvas de terceiro instar apresentam coloração cinza e quando estão terminando de completar esse instar adquirem coloração branca, o que caracteriza a fase de pré-pupa e passam posteriormente para a fase de pupa. A duração da fase de ovo a adulto de $P$. fulva é em média de 309,3 dias, o que caracteriza que a espécie pode formar uma geração por ano (univoltina).

Quando a larva de P. fulva vai atingir a fase de pupa, confecciona no fundo do frasco uma câmara, onde permanece abrigada até atingir a fase adulta. Rodrigues et al. (2008a) observaram esse mesmo comportamento para A. testaceipennis criado em laboratório.

Pelidnota virescens teve duração de primeiro instar de 15 dias, o segundo de 45 e o terceiro de 200 e a pupa de 29 dias (Morón \& Deloya 2002). Segundo Hayes (1925) P. punctata possui duração larval de 645 dias, para completar seu desenvolvimento, enquanto que a fase de pupa dessa mesma espécie segundo Hoffmann (1936) dura cerca 21,8 dias. O ciclo de $P$. fulva é semelhante ao de $P$. virescens, porém o ciclo de $P$. punctata é de cerca de dois anos.

As cápsulas cefálicas de primeiro, segundo e terceiro instar de P. fulva mediram respectivamente, 2,20, 4,10 e 6,54 mm. Morón \& Deloya (2002) verificaram para $P$. virescens que as larvas de primeiro instar mediram $2,25 \mathrm{~mm}$, as de segundo instar $3,8 \mathrm{~mm}$ e de terceiro instar 6,3 mm, apresentando, portanto, dimensões semelhantes.

A criação de $P$. fulva em laboratório utilizando-se de solo, material vegetal em decomposição e húmus foi adequado, com viabilidade de $48,8 \%$. Em criações de Scarabaeidae fitófagos em laboratório foram obtidas menores viabilidades como por Rodrigues et al. (2008a, b) para L. fuscus de $10,9 \%$ e A. testaceipennis, $16,3 \%$.

Ao atingir a fase adulta, $P$. fulva durou em média 12,8 dias (Tabela 1), apresentando assim, curto período de vida no laboratório.
Tabela 1. Duração (média $\pm \mathrm{EP}$ ) das fases de desenvolvimento de Pelidnota fulva Blanchard, 1850 em laboratório $\left(26 \pm 1^{\circ} \mathrm{C}\right.$, fotofase de 12 horas $)$.

Table 1. Duration (mean $\pm \mathrm{SE}$ ) of the development stage Pelidnota fulva Blanchard, 1850 under laboratory conditions $\left(26 \pm 1{ }^{\circ} \mathrm{C}\right.$, photoperiod of 12 hours).

\begin{tabular}{lcccc}
\hline \multicolumn{1}{c}{ Fase } & $\begin{array}{c}\text { Duração } \\
\text { (dias) }\end{array}$ & $\mathbf{N}$ & $\begin{array}{c}\text { Intervalo de } \\
\text { variação }\end{array}$ & $\begin{array}{c}\text { Viabilidade } \\
(\%)\end{array}$ \\
\hline Ovo & $15,1 \pm 1,13$ & 41 & $8-26$ & 100 \\
$1^{\circ}$ ínstar & $22,4 \pm 2,19$ & 41 & $7-55$ & 97,6 \\
$2^{\circ}$ ínstar & $36,9 \pm 1,78$ & 40 & $26-56$ & 72,5 \\
$3^{\circ}$ ínstar & $209,7 \pm 13,04$ & 29 & $162-241$ & 68,9 \\
Pupa & $20,7 \pm 0,49$ & 20 & $19-22$ & 100 \\
Adulto & $12,8 \pm 1,01$ & 20 & $10-17$ & 100 \\
Ovo a adulto & $309,3 \pm 13,66$ & 20 & $264-341$ & 48,8 \\
\hline
\end{tabular}

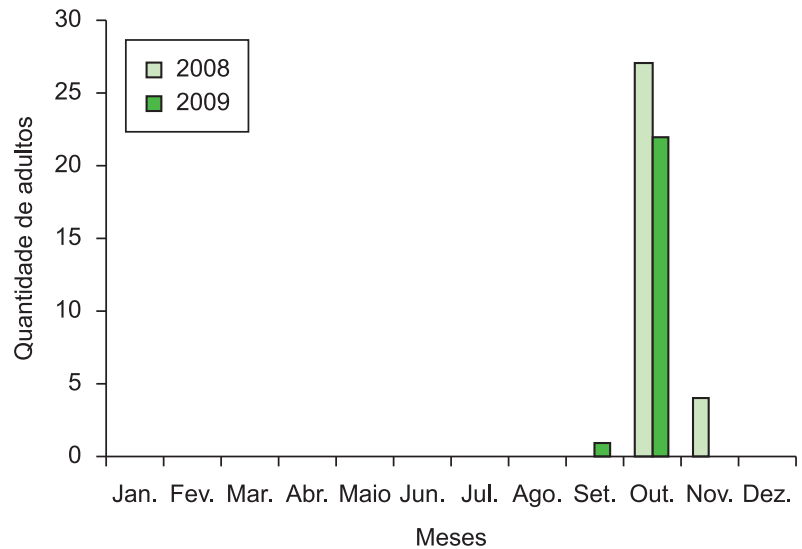

Figura 1. Quantidade de adultos de Pelidnota fulva Blanchard, 1850 coletados com armadilha luminosa e em fontes luminosas de janeiro de 2008 a dezembro de 2009, em Aquidauana, MS.

Figure 1. Number of adults Pelidnota fulva Blanchard, 1850 collected in traps and sources light from January 2008 to December 2009 in Aquidauana, MS.

\section{Agradecimentos}

Ao prof. Dr. Sérgio Ide do Instituto Biológico de São Paulo, pela identificação da espécie.

\section{Referências}

BIONE, E., MOURA, R.C., CARVALHO, R. \& SOUZA, M.J. 2005. Karyotype, C-and fluorescence banding pattern, NOR location and FISH study of five Scarabaeidae (Coleoptera) species. Genet. Mol. Biol. 28(3):376-381.

BLACKWELDER, R.E. 1944. Checklist of the coleopterous insetcs of México, Central America, The West Indies, and South America. Unit. Stat. Nat. Mus. Bull. 185(2):197-265.

BUSS, E.A. 2006. Flignt activity and relative abundance of phytophagous scarabs (Coleoptera: Scarabaeoidea) from two locations in Florida. Fl. Entomol. 89(1):32-40.

CARRILO RUIZ, H. \& MORÓN, M.A. 2003. Fauna de Coleoptera Scarabaeoidea de Cuetzalan de Progreso, Puebla, México. Act. Zool. Mex. 88:87-121.

CARVALHO, A.M.C. 2009. Guilda de abelhas e outros visitantes de Matayba guianensis (Sapindaceae) em vegetação de cerrado. Tese de Doutorado, Universidade Federal de Uberlândia, Uberlândia.

DELGADO-CASTILlO, L., DELOYA, G. \& MORÓN, M.A. 1988. Descripcion de dos nuevas especies mexicanas de Pelidnota (Coleoptera: Melolonthidae; Rutelinae). Fol. Entomol. Mex. (74):131-144. 
FLORES, C.P., RAMIREZ, A.E.C., MORÓN, M.A. \& GÓMEZ, B.G.Y. 2008. Fauna de escarabajos melolóntidos (Coleoptera: Scarabaeoidea) en el municipio de Villaflores, Chiapas, México. Act. Zool. Mex. 24:139-168.

FREITAS, F.A., ZANUNCIO, T.V., LACERDA, M.C. \& ZANUNCIO, J.C. 2002. Fauna de Coleoptera coletada com armadilhas luminosas em plantio de Eucalyptus grandis em Santa Bárbara, Minas Gerais. Rev. Árv. 26(4):505-511.

GARCIA, A.A., GARCIA, G.A.L., OLIVAS, A.R., ALVAREZ, P.C., COTA, J.R.V. \& MORÓN, M.A. 2009. Huéspedes vegetales de adultos de Coleopotera Scarabaeoidea en el Vale de Carrizo, Sinaloa, México. Soutwest. Entomol. 35(1):99-108.

HARDY, A.R. 1975. A revision of the genus Pelidnota of America North of Panamá (Coleoptera: Scarabaeidae: Rutelinae). Univ. Calif. Public. Entomol. 78(1):1-43.

HAYES, W.P. 1925. A comparative study of the history of certain phytophagous scarabaeid beetles. Kans. Agricult. Exp. Stat. Tech. Bull. 16(1):1-146.

HOFFMANN, C.H. 1936. Additions to our knowledge of the biology of Pelidnota punctata Linn. (Scarabaeidae - Coleoptera). J. Kan. Entomol. Soc. 9(3):103-105.

HOWDEN, H.F. 1998. A new species of Pelidnota Macleay from Chiriqui Province, Panama (Scarabaeidae, Rutelinae). Coleop. Bull. 52(2):171-173.

LORDELLO, L.G.E. 1951. Algumas notas sobre Macrodactylus pumilio Burm., 1855 e Pelidnota (Pelidnota) pallidipenns Bates, 1904 (Coleoptera, Scarabaeidae). Rev. Agric. 26:109-116.

MAES, J.M., RATCLIFFE, B.C. \& JAMESON, M.L. 1997. Fauna entomologica de la reserve natural Bosawas, Nicaragua. XI. Escarabajos (Coleoptera: Scarabaeidae) nuevos para la fauna de Nicarágua. Rev. Nicar. Ent. 39:41-46.

MORÓN, M.A. 1976. Description de las larvas de tres especies mexicanas de pelidnotinos (Coleopotera; Melolonthidae, Rutelinae), y algunas observaciones sobre su biologia. Anal. Inst. Biol. Série Zoológica. 47(1):7-17.
MORÓN, M.A. \& DELOYA, C. 2002. Observaciones sobre el ciclo de vida de Pelidnota (Pelidnota) virescens Burmeister, 1844 (Coleoptera: Melolonthidae: Rutelinae). Act. Zool. Mex. 85:109-118.

NEITA, J.C., OROZCO, J. \& RATCLIFFE, B. 2006. Escarabajos (Scarabaeidae: Pleurosticti) de la selva baja del Bosque Pluvial Tropical "BP-T", Chocó, Colombia. Act. Zool. Mex. 22:1-32.

PARDO-LOCARNO, L.C., MONTOYA-LERMA, J., BELLOTTI, A.C. \& SCHOONHOVEN, A.V. 2005. Structure and composition of the white grub complex (Coleoptera: Scarabaeidae) in: agroecological systems of Northern Cauca, Colombia. Fl. Entomol. 88(4):355-363.

PAUCAR-CABRERA. A. 2005. A catalog and distributional analysis of Rutelinae (Coleoptera: Scrabaeidae) of Ecuador. Zoot. 948:1-92.

RATCLIFFE, B.C. 2002. A checklist of the Scarabaeoidea (Coleoptera) of Panamá. Zootaxa. 32:1-48.

RODRIGUES, S.R., PUKER, A., ABOT, A.R., BARBOSA, C.L., IDE, S. \& COUTINHO, G.V. 2008a. Ocorrência e aspectos biológicos de Anomala testaceipennis Blanchard (Coleoptera, Scarabaeidae). Rev. Bras. Entomol. 52(1):68-71.

RODRIGUES, S.R., BARBOSA, C.L., PUKER, A., ABOT, A.R. \& IDE, S. 2008b. Occurrence, biology and behavior of Liogenys fuscus Blanchard (Insecta, Coleoptera, Scarabaeidae) in Aquidauana, Mato Grosso do Sul, Brazil. Rev. Bras. Entomol. 52(4):637-640.

RODRIGUES, S.R., NOGUEIRA, G.A.L., ECHEVERRIA, R.R. \& OLIVEIRA, V.S. 2010a. Aspectos biológicos de Cyclocephala verticalis Burmeister (Coleooptera: Scarabaeidae). Neotrop. Entomol. 39(1):15-18.

RODRIGUES, S.R., PUKER, A. \& TIAGO, E.F. 2010b. Aspectos biológicos de Leucothyreus dorsalis Blanchard (Coleoptera, Scarabaeidae, Rutelinae). Rev.Bras. Entomol. 54(3):431-435.

SILVA, A.G.A., GONÇALVES, C.R., GALVÃO, D.M., GONÇALVES, A.J.L., GOMES, J., SILVA, M.N. \& SIMONI, L. 1968. Quarto catálogo e os insetos que vivem nas plantas do Brasil. Seus parasitos e predadores. Lab. Central Patologia Vegetal. Ministério da Agricultura, Rio de Janeiro, Tomo 1, Parte II.

VIANA, C.H.P., MARQUES, O.M. \& CARVALHO, C.A.L. 2001. Rutelinae (Coleoptera: Scarabaeidae) coletados em armadilha luminosa em Cruz das Almas, Bahia. Magistra 13(1):9-13. 